\title{
Importanza di un impianto di fitodepurazione per l'avifauna acquatica nidificante e svernante. Il caso di una Cassa di colmata (Mira, Venezia, Italia settentrionale)
}

\author{
Francesco Scarton ${ }^{1 *}$, Mauro Bon ${ }^{2}$, Oliviero Trebbi $^{3}$
}

\begin{abstract}
Importance of a phytodepuration plant for the nesting and wintering aquatic avifauna. The case of a "Cassa di colmata" (Mira, Venice, northern Italy).

Results of field surveys of waterbirds made between 2011 and 2016 in a 110-ha constructed wetland for wastewater treatment indicate that eighteen waterbird species have nested. The most abundant were coot (up to 30-50 pairs in a single year), little grebe (40), purple heron (20), moorhen (10-20), mallard (10+), great crested grebe (3-12), and mute swan (2-8). Breeding species of environmental concern, rare in the whole Veneto region, include a few pairs of bittern, red-crested pochard and ferruginous duck. About 1,900 birds occurred on average in mid-January, with teal and mallard being the most common. The regular occurrence of the ferruginous duck and bittern is noteworthy. The constructed wetland proved valuable for waterbirds a few years after construction.
\end{abstract}

Key words: man made sites, bittern, ferruginous duck, purple heron, Venice lagoon.

Riassunto - Nell'impianto di fitodepurazione della Cassa di Colmata A (Mira, Venezia), ampia 110 ha di cui due terzi rappresentati da canneti, durante gli anni 2011-2016 è stata accertata la nidificazione di 17 specie di uccelli acquatici, sia comuni (cigno reale, folaga, tuffetto, germano reale) che di maggior interesse conservazionistico (tarabuso, moretta tabaccata, fistione turco). L'avifauna acquatica svernante include 29 specie; le più abbondati sono risultate germano reale e alzavola, con presenze anche di specie di interesse conservazionistico come moretta tabaccata e tarabuso. Come altri bacini di fitodepurazione, anche quello studiato ha assunto rilevante valore per l'avifauna selvatica nel volgere di un breve periodo di tempo dall'avvio del progetto.

Parole chiave: zone umide artificiali, tarabuso, airone rosso, moretta tabaccata

\footnotetext{
${ }^{1}$ Via R. Franchetti 192, 31022 Preganziol (Tv), Italia.

${ }^{2}$ Museo di Storia Naturale di Venezia. Fontego dei Turchi 1730, 30135 Santa Croce, Venezia, Italia.

E-mail: mauro.bon@fmcvenezia.it

${ }^{3}$ Via C. Monteverdi 11/B, Mestre (Ve), Italia.

E mail: oliviero.trebbi@libero.it

* Corrisponding author: scarton@selc.it

(C) 2017 Scarton, Mauro Bon, Oliviero Trebbi
}

Received: 26 October 2016

Accepted for pubblication: 5 June 2017

\section{INTRODUZIONE}

Una tecnica utilizzata da alcuni decenni a livello mondiale per il trattamento delle acque reflue consiste nell'invasare i liquami, generalmente già pretrattati con metodiche convenzionali, in bacini aventi modesta profondità e abbondante vegetazione acquatica, sia sommersa che emersa. I processi fisici e biologici che avvengono nelle aree vegetate, quali ad esempio filtrazione, adsorbimento, assimilazione da parte delle diverse specie di piante e degradazione batterica contribuiscono alla significativa riduzione del carico inquinante; numerose esperienze dimostrano come si possano raggiungere abbattimenti superiori all' $80 \%$ per i nutrienti, solidi sospesi e $\mathrm{BOD}_{5}$ (ANPA, 2002; ISPRA, 2012).

I bacini di fitodepurazione, specie quelli di dimensioni medio-grandi, possono ospitare in breve tempo un'abbondante fauna selvatica, sia acquatica che terrestre; gli uccelli acquatici ne costituiscono una componente molto rilevante, oggetto all'estero di numerosi studi, riassunti ad esempio da Hansson et al. (2005) e Murray \& Hamilton (2010).

In Italia l'avifauna acquatica presente nei bacini di fitodepurazione non sembra invece aver ottenuto grande attenzione, nonostante non manchino alcuni articoli apparsi su riviste ornitologiche (Angeletti et al., 2005; Giannella et al., 2013; Giannella \& Gemmato, 2014; Viganò et al., 2009).

Nel Veneto vi sono numerosi impianti di fitodepurazione, generalmente di tipo a flusso sub-superficiale e quindi di piccole o piccolissime dimensioni (Veneto Agricoltura, 2014). Tra quelli invece a flusso superficiale, di dimensione più ampia, i più significativi esempi sono il bacino delle Cave di Salzano (Venezia), che viene utilizzato anche per ridurre il carico inquinante delle acque sversanti nel bacino scolante della laguna di Venezia; l'impianto di Castelnovo Bariano (Rovigo), di 16 ha e realizzato alla fine degli anni Novanta lungo il Po su terreni golenali precedentemente utilizzati come pioppeto (ANPA, 2002); l'impianto di Ca' di Mezzo a Codevigo (Padova), esteso per 30 ha su preesistenti superfici agrarie ed oggetto di un dettagliato monitoraggio chimico-fisico delle acque (ANPA, 2002). Non sono invece disponibili informazioni di pari approfondimento sull'avifauna di questi siti, che pure viene genericamente definita come abbondante $\mathrm{o}$ interessante. 

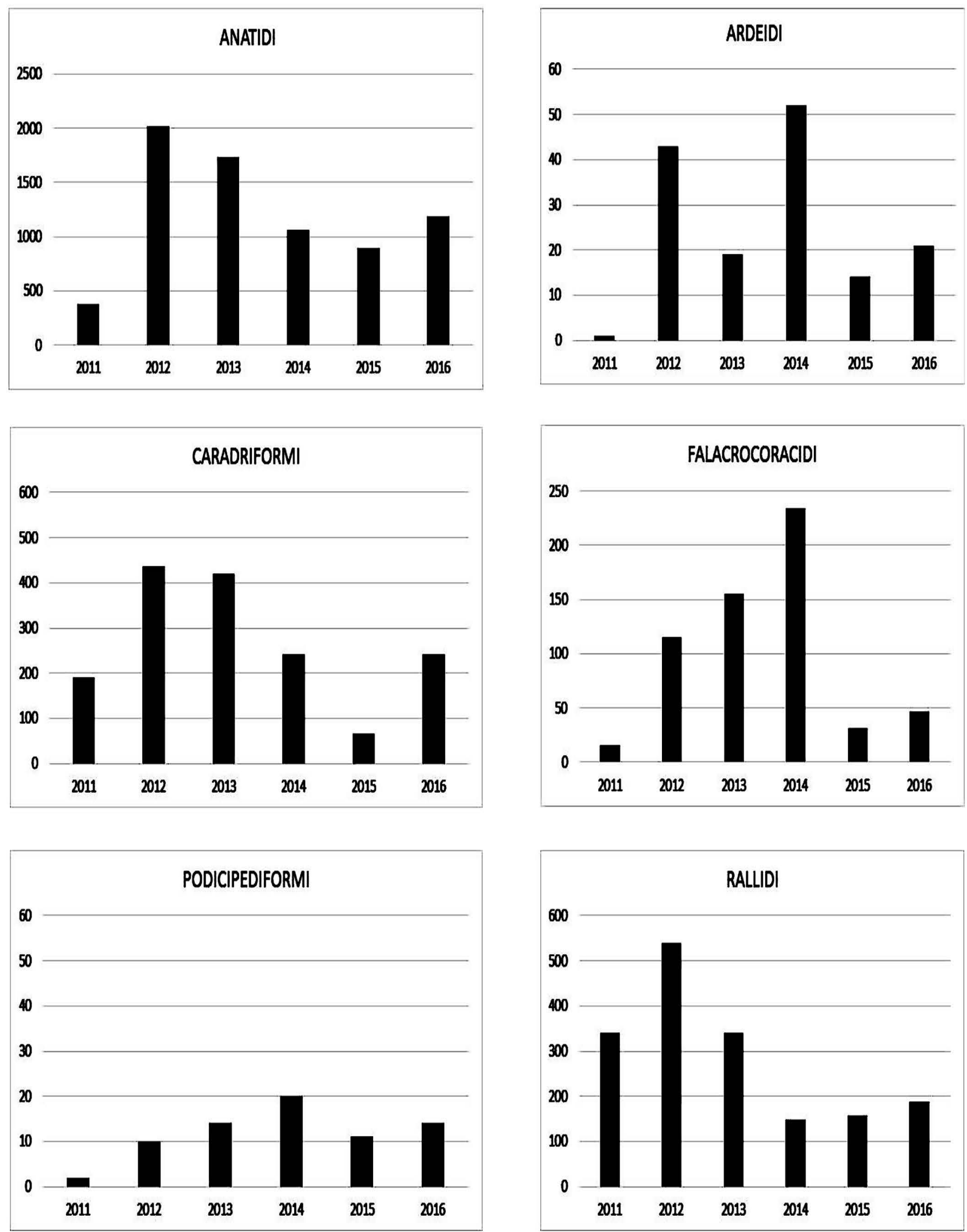

Fig. 1 - Presenze di avifauna acquatica a metà gennaio (dati rielaborati da Basso \& Bon, 2011-2016). / Waterbird occurrence during the mid-January counts (from Basso \& Bon, 2011-2016, modified). 
Riteniamo utile esporre una sintesi dei dati acquisiti nel 2011-2016 e relativi all'avifauna acquatica, nidificante e svernante, presente in un vasto e recente impianto di fitodepurazione a flusso superficiale, realizzato lungo il margine della laguna di Venezia e noto come "Cassa di colmata A".

\section{AREA DI STUDIO}

La Cassa di colmata A, estesa per circa 140 ha e con altimetria media di $1,5 \mathrm{~m}$ s.l.m. (coordinate geografiche: $45.4192^{\circ} \mathrm{N}, 12.2418^{\circ} \mathrm{E}$, comune di Mira) è un'isola artificiale realizzata come le vicine e più estese casse di colmata B e D/E negli anni Sessanta del secolo scorso, per colmamento di un'area lagunare a basso fondale con i fanghi provenienti dai lavori di scavo del canale lagunare Malamocco-Marghera. Lo scopo era la formazione di un'area dove sarebbe dovuta sorgere la terza zona industriale di Porto Marghera. A seguito del mutamento degli scenari ambientali e socio-economici dell'area lagunare, e alla mancata realizzazione della terza area industriale, le tre casse di colmata non furono tuttavia mai utilizzate per tale scopo e con il tempo subirono un fenomeno di spontanea rinaturalizzazione, che le hanno portate a divenire luoghi di estremo interesse per la fauna (Scarton et al., 2000; Roccaforte e Maschietto, 2002; Bon et al., 2014).

Alla metà del Duemila venne avviato dalla Regione Veneto il Progetto Integrato Fusina, che prevedeva tra le altre attività di destinare la Cassa di colmata $\mathrm{A}$ alla fitodepurazione di reflui di origine civile, prima del loro invio a riutilizzo duale-industriale, nonché alla funzione d'invaso delle acque in condizione di eventi piovosi rilevanti. Nella Cassa di colmata A vennero quindi scavati tre grandi bacini, separati da arginature e interconnessi tramite chiaviche, con profondità compresa tra 30 e 160 $\mathrm{cm}$ e superficie complessiva di 110 ettari; successivamente vennero messi a dimora circa 700.000 rizomi di specie igrofile (Regione del Veneto, 2016). L'impianto a flusso superficiale della Cassa di colmata A costituisce il più grande sito della sua categoria in Italia e probabilmente in tutt'Europa.

Circa due terzi dell'intera estensione, quindi $73 \mathrm{ha}$, risultano attualmente coperti da vegetazione acquatica, sia radicata (Phragmites communis soprattutto, secondariamente Typha latifolia e Schoenoplectus lacustris) oltre che flottante (Nymphea alba, Ceratophyllum demersum); le rimanenti estensioni, circa 37 ha, sono rappresentate da specchi d'acqua libera. Al margine settentrionale della Cassa di colmata A sono inoltre presenti alcuni nuclei, preesistenti alla realizzazione del bacino di fitodepurazione, di specie arboree come Populus alba e Salix alba.

I reflui provenienti dall'abitato di Marghera vengono prima trattati in un impianto convenzionale e successivamente entrano nell'impianto della Cassa di colmata A; le portate di ingresso e di uscita sono di $4.000 \mathrm{~m}^{3} / \mathrm{h}$. Una viabilità impostata sulla sommità degli argini perimetrali ed interni permette un facile spostamento agli automezzi di servizio; l'accesso all'area è ristretto al personale tecnico incaricato della gestione e controllo dell'impianto (Regione Veneto, 2016).

\section{MATERIALI E METODI}

Per l'avifauna acquatica si sono considerati in questo lavoro due periodi, vale a dire quello della nidificazione (aprile-luglio) e dello svernamento (dicembre-gennaio). I dati sono stati raccolti sia in modo opportunistico, durante frequenti visite effettuate nell'arco dell'anno da O.T., che con uscite volte alla stima delle coppie nidificanti ed effettuate ogni anno dai tre Autori nel periodo 20 maggio - 10 giugno del 2011-2016. Per l'avifauna svernante sono stati utilizzati i dati provenienti dai censimenti IWC di metà gennaio, anni 2011-2016, eseguiti dalla Provincia di Venezia e pubblicati in relazioni tecniche inedite (Basso \& Bon, 2011-2016). L'ordine sistematico e la nomenclatura scientifica sono quelli riportati da Brichetti \& Fracasso (2015).

\section{RISULTATI}

Complessivamente hanno nidificato 17 specie di uccelli acquatici, elencate in Tabella 1. Il totale annuale delle coppie nidificanti presenta forti variazioni, dovute in gran parte al germano reale Anas platyrhynchos. Si precisa tuttavia che per questa specie i valori riportati sono indicativi; oltre ai riproduttori, vi erano ogni anno circa 300 soggetti non nidificanti, che utilizzavano la Cassa di colmata A come area di muta e la cui presenza ha complicato le stime qui esposte.

Nel 2013 e nel 2014 è stata osservata una coppia di svasso piccolo Podiceps nigricollis, ma non vi sono riscontri di una sua nidificazione e pertanto la specie non viene riportata in Tabella 1. Accertata invece, sebbene non regolarmente, la riproduzione di due specie di elevato interesse conservazionistico come fistione turco Netta rufina e moretta tabaccata Aythya niroca. Fino a 150 individui di moriglione Aythya ferina sono stati osservati in periodo riproduttivo, ma le coppie nidificanti sono state solo 1-3, in quasi tutti gli anni di indagine.

Tra gli Ardeidi spicca la presenza di tre adulti di tarabuso Botaurus stellaris nel giugno 2012; riteniamo che in quell'anno fosse presente almeno una coppia di questa rara specie. Mai osservate nidificazioni di Phalacrocoracidae, sebbene i nuclei alberati della Cassa di colmata A fossero regolarmente utilizzati per la sosta diurna da un massimo di 300 marangoni minori Microcarbo pygmeus e da 30-50 cormorani Phalacrocorax carbo. Non sono stati osservati Caradriformi nidificanti, data l'assenza di superfici idonee per la realizzazione del nido.

Le specie presenti durante i censimenti IWC di metà gennaio 2011-2016 sono state 15-21 (Basso \& Bon, 2011-2016); la media per ogni anno è stata di 1904 uccelli $($ d.s. $=+864,3)$, in gran parte Anatidi $(1210,8)$, Rallidi $(285,7)$ e Laridi $(265,7)$. Il numero di specie aumenta con gli anni, cosi come la diversità del popolamento svernante, mentre il numero di individui diminuisce. Dopo un repentino incremento nei primi due anni il totale degli svernanti è in calo fino al 2015, con un lieve recupero nel 2016 (Fig. 1); le forti variazioni sono addebitabili quasi esclusivamente all'alzavola Anas crecca ed al germano reale, le due specie più abbondanti con 
Tab. 1 - Elenco delle specie di uccelli acquatici nidificanti e numero minimo di coppie stimato. / Nesting waterbird species and minimum estimated number of pairs.

\begin{tabular}{|c|c|c|c|c|c|c|c|c|}
\hline & 2011 & 2012 & 2013 & 2014 & 2015 & 2016 & Media & d.s. \\
\hline Cygnus olor & 0 & 8 & 2 & 4 & 6 & 2 & 3,7 & 2,9 \\
\hline Anas strepera & 0 & 3 & 1 & 3 & 3 & 1 & 1,8 & 1,3 \\
\hline Anas platyrhynchos & 4 & 60 & 80 & 30 & 80 & 60 & 52,3 & 29,9 \\
\hline Anas clypeata & 0 & 1 & 3 & 1 & 0 & 0 & 0,8 & 1,2 \\
\hline Anas querquedula & 0 & 0 & 0 & 2 & 0 & 2 & 0,7 & 1,0 \\
\hline Anas crecca & 0 & 1 & 0 & 0 & 0 & 0 & 0,2 & 0,4 \\
\hline Netta rufina & 1 & 0 & 0 & 0 & 3 & 3 & 1,2 & 1,5 \\
\hline Aythya ferina & 1 & 2 & 2 & 2 & 3 & 1 & 1,8 & 0,8 \\
\hline Aythya nyroca & 0 & 0 & 0 & 2 & 1 & 1 & 0,7 & 0,8 \\
\hline Tachybaptus ruficollis & 20 & 40 & 12 & 15 & 35 & 8 & 21,7 & 13,0 \\
\hline Podiceps cristatus & 3 & 3 & 4 & 6 & 12 & 6 & 5,7 & 3,4 \\
\hline Botaurus stellaris & 0 & 1 & 0 & 0 & 0 & 0 & 0,2 & 0,4 \\
\hline Ixobrychus minutus & 1 & 2 & 0 & 1 & 0 & 0 & 0,7 & 0,8 \\
\hline Ardea cinerea & 0 & 1 & 1 & 3 & 4 & 3 & 2,0 & 1,5 \\
\hline Ardea purpurea & 10 & 15 & 10 & 15 & 20 & 10 & 13,3 & 4,1 \\
\hline Gallinula chloropus & 1 & 1 & 1 & 0 & 1 & 2 & 1,0 & 0,6 \\
\hline Fulica atra & 10 & 50 & 15 & 30 & 20 & 15 & 23,3 & 14,7 \\
\hline Numero specie & 9 & 14 & 11 & 13 & 12 & 13 & 12,0 & 1,8 \\
\hline Totale coppie & 51 & 188 & 131 & 114 & 188 & 114 & 131,0 & 51,9 \\
\hline
\end{tabular}

una media annuale di circa 490 individui ciascuna. Le altre specie più abbondanti sono folaga Fulica atra $(280$ individui) e gabbiano reale Larus michahellis (172); di rilievo anche la presenza di moriglione Aythya ferina (113), marangone minore (87), mestolone Anas clypeata (78) e moretta tabaccata, quest'ultima con media di 4 e massimo di 12 individui nel 2012. Le osservazioni condotte al di fuori dei censimenti di metà gennaio evidenziano la regolare presenza del tarabuso, fino a 3 individui osservati nello stesso giorno.

\section{DISCUSSIONE E CONCLUSIONI}

Le osservazioni condotte per sei anni evidenziano l'importanza che il bacino di fitodepurazione della Cassa di colmata A ha acquisito per l'avifauna acquatica. Tra 9 e 14 specie si sono riprodotte ogni anno; alcune sono di interesse conservazionistico e rare nell'intero Veneto. Per tarabuso, moretta tabaccata e fistione turco la Cassa di colmata A è uno dei pochissimi siti regionali in cui la nidificazione sia considerata probabile o certa (Mezzavilla et al., 2016). Per lo svasso maggiore Podiceps cri- 
status il bacino di fitodepurazione costituisce invece il sito dove si concentra il maggior numero di coppie della provincia di Venezia; la presenza numericamente significativa della folaga è in accordo con quanto osservato da Viganò et al. (2009) in un bacino di fitodepurazione lombardo.

L'insediamento di una colonia di airone rosso Ardea purpurea dimostra il valore che i canneti degli impianti di fitodepurazione possono avere per questa specie; attualmente l'aione rosso appare in preoccupante declino in tutta la laguna di Venezia (Scarton \& Valle, 2017) per cause che non sono note. La presenza di fitti canneti, in aree con limitato disturbo antropico come quella in esame, può costituire un elemento di rilievo per la conservazione dell'airone rosso, che tende ad evitare per la nidificazione le aree umide prossime a possibili sorgenti di disturbo quali strade e nuclei abitati (Kazantzidis et al., 2013).

Piuttosto elevate le presenze di uccelli nella Cassa di colmata $\mathrm{A}$ anche durante lo svernamento; a metà gennaio il popolamento di uccelli acquatici è dominato da due anatidi, alzavola e germano reale, le cui ampie fluttuazioni interannuali sono di difficile interpretazione. Il progressivo calo degli svernanti è probabilmente da mettere in relazione con l'aumento delle superfici vegetate rispetto a quelle ad acqua libera.

Lo svernamento regolare della moretta tabaccata, che viene mediamente censita con meno di dieci individui nell'intera laguna di Venezia (Bon \& Scarton, 2012), è altro elemento di interesse; l'importanza che in Italia i bacini di fitodepurazione possono avere per questa specie era già stata evidenziata nel relativo Piano d'Azione Nazionale (Melega, 2007). Anche la regolare presenza invernale del tarabuso e la sua probabile nidificazione in almeno uno degli anni di studio evidenzia il valore che le zone umide artificiali possono acquisire per questa specie, come già rilevato per altri bacini di fitodepurazione in Italia (Giannella \& Gemmato, 2014); nel Regno Unito estesi programmi di creazione di nuove zone umide sono stati intrapresi per favorire la presenza del tarabuso (Gilbert et $a l ., 2005)$. Considerato il valore dell'impianto di fitodepurazione della Cassa di colmata A per l'avifauna, e per la fauna più in generale, auspichiamo che possa essere nel prossimo futuro destinato anche alle visite naturalistiche e allo svolgimento di indagini faunistiche.

\section{Ringraziamenti}

Si ringrazia la società SIFA S.C.p.A. per aver cortesemente concesso l'ingresso all'impianto.

\section{BIBLIOGRAFIA}

Angeletti G., Brescia E., Gambeli P., Politi P. \& Sebastianelli C., 2005 - Comunità ornitica svernante nell'impianto di fitodepurazione di Jesi (Ancona). In: Atti XII Convegno Italiano di Ornitologia. Boano G. Cuccco M., Pavia M. \& Rubolini D. (eds.). Avocetta, 29: 43.

ANPA-Agenzia Nazionale per la Protezione Ambientale, 2002 - Linee guida per la ricostruzione di aree umide per il trattamento di acque superficiali Manuali e linee guida $9 / 2002$.
Basso M. \& Bon M., 2011-2016 - Censimento degli uccelli acquatici svernanti sul territorio della Provincia di Venezia, gennaio 2011-2016. Provincia di Venezia. Servizio Caccia e Pesca. Relazioni non pubblicate.

Bon M. \& Scarton F., 2012 - Lo svernamento degli uccelli acquatici in provincia di Venezia (1993-2012). Provincia di Venezia - Assessorato alla caccia.

Bon M., Scarton F., Stival E., Sattin L. \& Sgorlon G., 2014 - Nuovo Atlante degli uccelli nidificanti e svernanti in provincia di Venezia. Associazione Faunisti Veneti, Museo di Storia Naturale di Venezia.

Brichetti P. \& Fracasso G., 2015 - Check-list degli uccelli italiani aggiornata al 2014. Rivista Italiana di Ornitologia. Research in Ornithology, 85: 31-50.

Giannella C., Baroni G., Tosatti G. \& Tosatti L., 2013 Time-budget per alcune specie di Anatidae in migrazione prenuziale nella bassa Modenese. Rivista Italiana di Ornitologia, 82: 232-235.

Giannella C. \& Gemmato R., 2014 - Risultati di tre anni (2009-2011) di monitoraggio nella Zona a Protezione Speciale 404018 Le Meleghine. In: Atti XVI Convegno Italiano di Ornitologia. Cervia (RA), 22-25 settembre 2011. Tinarelli R., Andreotti A., Baccetti N., Melega L., Roscelli F., Serra L. \& Zenatello M. (a cura di). Scritti, Studi e Ricerche di Storia Naturale della Repubblica di San Marino: 177-178.

Gilbert G., Tyler G.A., Dunn C.J. \& Smith K.J., 2005 Nesting habitat selection by bitterns Botaurus stellaris in Britain and the implications for wetland management. Biological Conservation, 124: 547-553.

Hansson L. A., Brönmark C., Anders Nilsson P. \& Åbjörnsson K., 2005 - Conflicting demands on wetland ecosystem services: nutrient retention, biodiversity or both? Freshwater Biology, 50: 705-714.

ISPRA-Istituto Superiore per la Protezione e Ricerca Ambientale, 2012 - Guida tecnica per la progettazione e gestione dei sistemi di fitodepurazione per il trattamento delle acque reflue urbane. Manuali e linee guida, 12 .

Kazantzidis, S., Yfantis, G. \& Poirazidis K., 2013 - Factors influencing species composition and nest abundance of heron colonies. Journal of Biological Research, 20 (1): 276-289.

Melega L. (a cura di), 2007 - Piano d'azione nazionale per la Moretta tabaccata (Aythya nyroca). Quad. Cons. Natura, 25, Min. Ambiente - Ist. Naz. Fauna Selvatica.

Mezzavilla F., Scarton F. \& Bon M., 2016 - Uccelli del Veneto. Biologia, distribuzione, abbondanza. Zanetti $E d$.

Murray C. \& Hamilton A., 2010 - Perspectives on wastewater treatment wetlands and waterbird conservation. Journal of Applied Ecology, 47: 976-985.

Regione del Veneto, 2016 - Il Progetto Integrato Fusina e l'utilizzo della Fitodepurazione. $<$ http://sistemavenezia.regione.veneto.it/content/progetto-integrato-fusina $>$ (scaricato il 15/10/2016).

Roccaforte P. \& Maschietto G., 2002 - La Cassa di Colmata "A" della Laguna di Venezia. Gli Ambienti, la Flora, la Fauna. Oikos, Castelfranco Veneto (TV). 
Scarton F., Semenzato M., Tiloca G. \& Valle R. 2000 L'avifauna nidificante nelle casse di colmata B e D/E (non-Passeriformes): situazione al 1998 e variazioni intercorse negli ultimi venti anni. Bollettino del Museo Civico di Storia Naturale di Venezia, 50: 249-261.

Scarton F. \& Valle R., 2017- Andamento recente (20132015) delle popolazioni di uccelli acquatici nidificanti nella laguna aperta di Venezia. Bollettino del Museo Civico di Storia Naturale di Venezia, 67: 113-124.

Veneto Agricoltura, 2014 - La fitodepurazione per il trattamento di acque di origine agricola e di reflui zootecnici. Azienda Regionale per i Settori Agricolo, Forestale ed Agroalimentare.

Viganò A., Pellitteri-Rosa D., Vigorita V. \& Fasola M., 2009 - Zone umide artificiali: ciclo annuale dell'avifauna acquatica dell'Arnetta (Va-Mi). Alula, XVI: 814-816. 